Research Paper

\title{
Phthalocyanine-Aggregated Polymeric Nanoparticles as Tumor-Homing Near-Inirared Absorbers for Photothermal Therapy of Cancer
}

\section{Chang-Keun Lim¹, Jiyoung Shin², Yong-Deok Lee ${ }^{1}$, Jungahn Kim³ ${ }^{3}$ Keun Sang Oh${ }^{1}$, Soon Hong Yuk ${ }^{4}$, Seo Young Jeong'2, Ick Chan Kwon', Sehoon Kim ${ }^{1 凶}$}

1. Center for Theragnosis, Korea Institute of Science and Technology, 39-1 Hawolgok-dong, Seongbuk-gu, Seoul 136-791, Korea;

2. Department of Life and Nanophaceutical Science, Kyung Hee University, 1 Hoegi-dong, Dongdaemun-gu, Seoul 130-701, Korea;

3. Department of Chemistry, Kyung Hee University, Seoul 130-701, Korea;

4. College of Pharmacy, Korea University, Jochiwon, Yeongi, Chungnam, 339-700, Republic of Korea.

Corresponding author: Sehoon Kim, Center for Theragnosis, Korea Institute of Science and Technology, 39-1 Hawolgok-dong, Seongbuk-gu, Seoul 136-791, Korea. Tel: +82-2-958-5924; Fax: +82-2-958-5909; E-mail: sehoonkim@kist.re.kr.

(C) Ivyspring International Publisher. This is an open-access article distributed under the terms of the Creative Commons License (http://creativecommons.org/ licenses/by-nc-nd/3.0/). Reproduction is permitted for personal, noncommercial use, provided that the article is in whole, unmodified, and properly cited.

Received: 2012.01.22; Accepted: 2012.04.23; Published: 2012.09.20

\begin{abstract}
Phthalocyanine-aggregated Pluronic nanoparticles were constructed as a novel type of near-infrared (NIR) absorber for photothermal therapy. Tiny nanoparticles ( $60 \mathrm{~nm}$, FPc NPs) were prepared by aqueous dispersion of phthalocyanine-aggregated self-assembled nanodomains that were phase-separated from the melt mixture with Pluronic. Under NIR laser irradiation, FPc NPs manifested robust heat generation capability, superior to an individual cyanine dye and cyanine-aggregated nanoparticles. Micro- and macroscopic imaging experiments showed that FPc NPs are capable of internalization into live cancer cells as well as tumor accumulation when intravenously administered into living mice. It is shown here that continuous NIR irradiation of the tumor-targeted FPc NPs can cause phototherapeutic effects in vitro and in vivo through excessive local heating, demonstrating potential of phthalocyanine-aggregated nanoparticles as an all-organic NIR nanoabsorber for hyperthermia.
\end{abstract}

Key words: Phototermal therapy; Phthalocyanine; Near-infrared photonics; Tumor targeting; Hyperthermia; Nanoparticles.

\section{Introduction}

Organic dye-based near-infrared (NIR) photonics have widely been utilized in the fields of life and materials sciences. In biomedical applications, the utilization of dyes that absorb and fluoresce in the NIR range (ca. 600-1000 nm) takes an advantage of spectral coincidence with the tissue transparent window where minimal photon interferences (absorption, autofluorescence, and scattering) from solvents, biomolecules, and the biological matrix allow for deeper light penetration [1]. Promising applications include visualization of deep tissues by fluorescence imaging [2] and noninvasive treatment of deep tumors by photodynamic therapy (PDT) [3], both of which are attained by using NIR dyes that are fluorescent or capable of photosensitizing cytotoxic singlet oxygen $\left({ }^{1} \mathrm{O}_{2}\right)$, respectively. On the other hand, there has been a great deal of effort in the exploitation of organic dyes for use in high-density optical storage [4, 5]. Compact disc-recordable (CD-R) and digital versatile disc-recordable (DVD-R) are examples of NIR pho- 
tonics-based optical memory where micrometer-sized pits are written in the recording layer by the localized heat generation through the focused laser absorption-induced photothermal effect of NIR dyes [6-9].

Besides its application to optical devices, the photothermal effect of NIR dyes has been explored as a light-activated heat-generation modality for cancer hyperthermia $[10,11]$. It is known that protein denaturation, disruption of the cellular membrane and ablation of tumor tissues occur at temperatures of $40-43{ }^{\circ} \mathrm{C}$ or greater $[12,13]$. Selective photothermal therapy (PTT) is achieved by co-localization of laser irradiation and preferentially accumulated NIR dyes at tumor sites in which the photoexcited molecules are relaxed non-radiatively and thus the absorbed photon energy is converted into heat enough to induce cellular hyperthermia. In recent years, PTT has burgeoned into a promising niche of non- or minimally invasive tumor treatments, with the advent of inorganic nanomaterials including carbon nanotubes [14-17] and noble metal colloids. In particular, NIR-absorbing gold nanostructures (nanorods, nanoshells, nanocages, and hollow nanospheres) are well suited for PTT owing to the enhanced optical properties and high photothermal conversion efficiency as well as low toxicity [18-22].

Compared to inorganic counterparts, organic NIR dyes (mostly cyanines) possess advantageous features for biomedical applications, i.e., relatively high light absorptivity per mass, high-to-moderate fluorescence efficiency, and biocompatibility with less potential toxicity [23]. As for the PTT application, however, they present inherent limitations, such as low photothermal conversion efficiency due to competitive photophysical processes (ex. fluorescence or intersystem crossing), low photostability under intense and prolonged light irradiation, and overall, low efficiency of heating the surroundings for hyperthermia. To address these issues, we devised a new approach based on NIR dye aggregates that will be more suitable than using individual dye molecules for the application to PTT of cancer. The utilization of nanoscopic dye aggregates as a PTT agent will provide several advantages: (1) cumulatively enhanced light absorptivity due to the high chromophore density in the individual nano-agent, (2) enhanced photothermal conversion efficiency by aggregation-induced blocking of photophysical processes other than internal conversion and thermal relaxation, (3) high bioapplicability by forming surfactant-stabilized nanoparticles in aqueous physiological environment, and (4) possibility of passive tumor targeting by the enhanced permeability and retention (EPR) effect [24].

With these considerations in mind, we have constructed a new composite nanoformulation that is concentrated with photostable NIR dyes inside and surrounded with biocompatible polymer chains on the surface. As shown in Figure 1, the designed structure of dye-aggregated composite nanoparticles (FPc NPs) is based on the physical assembly of all organic constituents, i.e., tetra-t-butylphthalocyanine $\left(\mathrm{PcBu}_{4}\right)$ as a concentrated hydrophobic NIR dye and Pluronic F-68 as a biocompatible polymer surfactant, as well as glycol chitosan and heparin as particle surface adsorbents. Phthalocyanines are one of the most stable and tinctorially strongest chromophores having absorption in the NIR [25]. Owing to the outstanding optical and thermal stability compared to cyanines, they have widely been exploited as a photothermal-mode optical recording medium in a form of aggregated thin films [26]. Consequently, it is anticipated that Pluronic nanoparticles concentrated with a hydrophobic phthalocyanine will construct a dye-aggregated inner domain with favorable properties for the PTT activity in terms of NIR absorptivity, photothermal conversion efficiency, and stability. The hydrophilic polyethylene glycol (PEG) surface of the Pluronic-based nanoparticles is known to help prolonged blood circulation and hence EPR-effected tumor accumulation by reducing opsonization in the reticuloendothelial systems (RES) [27], as well as cellular uptake of nanoparticles through enhanced endocytosis [28, 29]. In addition, heparin on the particle surface can enhance the in vivo tumor targeting efficiency due to its specific interaction with a fibrinogen-derived product found in the solid tumor [30, 31]. Taken together, the designed nanoformulation is expected to offer an optimal platform for the enhanced therapeutic efficacy of PTT. In this study, we report the efficient tumor targeting behavior of FPc NPs in vivo, along with their robust photostability and photothermal effect superior to an individual dye molecule, indocyanine green (ICG) as well as the control nanoparticles concentrated with a general hydrophobic cyanine dye, 1,1',3,3,3',3'-hexamethylindotricarbocyanine iodide ( $\mathrm{IcMe}_{6}$, Figure 1). Through this phthalocyanine-aggregated nanocomposite approach, we demonstrate potential use of all-organic NIR nanoabsorbers for photothermal therapy of cancer in cells and in vivo.

\section{Experimental Section}

Instrumentation: Transmission electron microscopic (TEM) images of negatively stained particles (2 wt.\% uranyl acetate) were obtained with a CM30 electron microscope (FEI/Philips) operated at $200 \mathrm{kV}$. Zeta potential studies were performed with Zetasiser nano ZS (Malvern Instruments). Absorption and flu- 
orescence spectra were acquired using a UV-visible spectrometer (Agilent 8453) and a fluorescence spectrophotometer (Hitachi F-7000, wavelength calibrated for excitation and emission), respectively.

Preparation of FPc NPs and FIc NPs: $\mathrm{PcBu}_{4}$-loaded nanoparticles were prepared by a temperature-induced phase transition method [31]. First, $25 \mathrm{mg}$ of $\mathrm{PcBu}_{4}, 100 \mathrm{mg}$ of PEG (Mw. 400), and $400 \mathrm{mg}$ of Pluronic F-68 were mixed and then the mixture was melted into the liquid phase with increase of the temperature to $150{ }^{\circ} \mathrm{C}$ for $90 \mathrm{~min}$. Immediately after quenching in an ice bath, a waxy solid was obtained. Then Mili-Q water $(20 \mathrm{~mL})$ was added to $100 \mathrm{mg}$ of the solid and the mixture was vigorously stirred to afford an aqueous dispersion of $\mathrm{PcBu}_{4}$-loaded nanoparticles (bear FPc NPs). For the surface modification with glycol chitosan (GC) and Heparin (Hep), $2 \mathrm{~mL}$ of a $0.0125 \mathrm{wt} \%$ GC solution was added to form GC adsorbed surface by hydrogen bonding, followed by stirring at ambient temperature for $10 \mathrm{~min}$. To obtain the final product, $2 \mathrm{~mL}$ of $0.125 \mathrm{wt} \%$ Hep solution was added to the reaction mixture and then the colloidal suspension was freeze-dried to obtain a blue powder of FPc NPs. To utilize in vivo/vitro NIRF imaging, 0.4 $\mathrm{mg}$ of Cy5.5-vinylsulfone $\left(10 \mathrm{mg} \mathrm{mL}^{-1}\right.$, BioActs Co. Ltd.) was added to the GC-Hep modified colloidal suspension and the mixture was dialyzed against distilled water using a Cellu. Sep membrane (Membrane Filteration Products, Inc., molecular cutoff $=12,000$ $\sim 14,000)$. Then, the resulting dispersion of nanoparticles was freeze-dried to obtain a blue powder of Cy5.5 labeled FPc NPs.

Measurement of singlet oxygen generation quantum yield with RNO test: Singlet oxygen generation was monitored by a decrease in the absorbance of the mixed RNO at $440 \mathrm{~nm}$ under laser excitation. The mixture solution was prepared by mixing 0.2 $\mathrm{mL}$ of FPc NPs $(1 \mathrm{mg} \mathrm{mL}-1)$ in water with $0.11 \mathrm{~mL}$ of RNO stock solution ( $0.12 \mathrm{mM}$ in water), $0.7 \mathrm{~mL}$ of histidine $(0.03 \mathrm{M}$ in water) and $0.18 \mathrm{~mL}$ water. NIR excitation at $671 \mathrm{~nm}$ was performed by irradiating the collimated laser beam through the sample. The relative quantum yields of ${ }^{1} \mathrm{O}_{2}$ generation $\left(\Phi_{\Delta}\right)$ were estimated by using a water solution of $\mathrm{Ce} 6$ as a reference and comparing the derived slopes of RNO absorbance plots (-ln $\mathrm{A}_{440}$ ) against the irradiation time.

Temperature evaluation induced by laser irradiation: DPSS $671 \mathrm{~nm}$ laser (Shanghai Dream Laser Technology Co., Ltd.) with a center wavelength of 671 $\pm 1 \mathrm{~nm}$ and $200 \mathrm{~mW}$ output power. To evaluate the laser induced temperature increase, $20 \mu \mathrm{L}$ of FPc NPs and FIc NPs suspensions, and ICG aqueous solution were added into wells of micro plate, respectively. Then each well was irradiated with the laser for 15 min, while the temperature was measured by thermometer with a needle probe.

FPc NPs uptake and NIR fluorescence imaging with tumor cells: SCC7 (squamous cell carcinoma) cells were cultivated in RPMI1640 medium supplemented with $10 \% \mathrm{FBS}, 100 \mathrm{U} / \mathrm{mL}$ penicillin and 100 $\mathrm{mg} / \mathrm{mL}$ streptomycin, in a humidified $5 \% \mathrm{CO}_{2}$ incubator at $37{ }^{\circ} \mathrm{C}$. The tested cells were seeded onto 35 $\mathrm{mm}$ coverglass bottom dishes and allowed to grow until a confluence of $70 \%$. Prior to the experiment, cells were washed twice with PBS ( $\mathrm{pH} 7.4$ ) to remove the remnant growth medium, and then incubated in a serum-free medium $(2 \mathrm{~mL})$ containing FPc NPs $(0.2$ $\mathrm{mg}$ ) for $1 \mathrm{~h}$. The cells were then washed twice with ice-cold PBS ( $\mathrm{pH}$ 7.4) and directly imaged using a fluorescence microscope (Ziess Axioskop2 FS Plus) with a filter set of $600-650 \mathrm{~nm}$ for excitation and 670-720 $\mathrm{nm}$ for emission.

In Vivo NIRF imaging with tumor bearing mice: The animal studies have been approved by the animal care and use committee of Korea Institute of Science and Technology and all handling of mice was performed in accordance with the institutional regulations. Tumor xenografts were created via subcutaneous injection of $1 \times 107$ SCC7 cells suspended in RPMI1640 cell culture media in 5-week-old male BALB/c nude mice (Orient. Korea). After 2 weeks post-inoculation, the mice were intravenously injected with the FPc NPs suspension (0.1 mg FPc NPs in 100 $\mu \mathrm{L} \quad$ DPBS). Their tissue distribution and time-dependent excretion profiles in murine tumor model were imaged by positioning mice on an animal plate heated to $37{ }^{\circ} \mathrm{C}$ in the eXplore Optix System (Advanced Research Technologies Inc., Canada). Laser power and count time settings were optimized at 5 $\mu \mathrm{W}$ and $0.3 \mathrm{~s}$ per point. A 670 -nm pulsed laser diode was used to excite the nanoparticle.

Photothermal toxicity evaluation with MTT cell viability assay: SCC7 cells were cultured in a 96-well plate and then a half of the well treated with FPc NPs ( $1 \mathrm{mg}$ ) for $5 \mathrm{~h}$ and the other half of well was remained without treatment as a control. After washing with fresh media, the plate was incubated for $12 \mathrm{~h}$ after laser treatment $\left(671 \mathrm{~nm}, 6.4 \mathrm{~W} / \mathrm{cm}^{2}\right)$ with irradiation times of $0 \mathrm{~min}, 5 \mathrm{~min}, 10 \mathrm{~min}, 15 \mathrm{~min}$, and $20 \mathrm{~min}$, to determine phototoxicity. Then, the cell viability was evaluated via MTT assay.

Photothermal therapy of tumor-bearing mice: The prepared tumor xenografts models with and without intravenous injection of the FPc NPs suspension $(0.2 \mathrm{mg}$ FPc NPs in $200 \mu \mathrm{L}$ DPBS) were continuously scanned with a $671 \mathrm{~nm}$ laser (seven spots in the tumor tissue) for $21 \mathrm{~min}$ at $4 \mathrm{~h}$ post-injection. The tumor size of each mouse was measured with a digital 
caliper, and the tumor volume was calculated as width $\times$ length $\times$ height $\times 1 / 2$ for 13 days.

\section{Results and Discussion}

FPc NPs were fabricated by colloidal dispersion of self-assembled aromatic nanodomains in the F-68 matrix. The process involves 1) preparing a ternary mixture of F-68, $\mathrm{PcBu}_{4}$, and polyethylene glycol (PEG, $M_{n}=400$ ) in a weight ratio of 100:6.25:25, by hot mechanical stirring at $150{ }^{\circ} \mathrm{C}$ for $1.5 \mathrm{~h}, 2$ ) quenching the hot mixture in an ice bath to induce nanoscopically confined self-assembly of $\mathrm{PcBu}_{4}$ by phase separation from the polymer matrix, and 3) subsequently dispersing the self-assembled $\mathrm{PcBu}_{4}$ colloids by adding water to the quenched mixture. During the steps, the $\mathrm{PcBu}_{4}$-aggregated nanodomains were well-separated and stably dispersed in water by the surface stabilization behavior of a polymeric surfactant (F-68). The transmission electron microscopic (TEM) image elucidates that the obtained colloids are spherical nanoparticles with an average diameter of $75 \pm 12 \mathrm{~nm}$ (Figure 2A), which is appropriate for facile blood circulation and endocytosis of nanomaterials [32-34]. For a comparative study on the photothermal effect, control nanoparticles of non-phthalocyanine aggregates (FIc NPs) were formulated with a general cyanine dye $\left(\mathrm{IcMe}_{6}\right)$. Owing to the low thermal stability of cya- nines, hot mechanical mixing was not able to be processed for the phase-separated dye assembly. Instead, the preparation process was modified into solution mixing of $\mathrm{IcMe}_{6}$ with a more hydrophobic Pluronic F-127 and then colloidal dispersion by adding water to the dried mixture, to yield cyanine-aggregated nanoparticles (FIc NPs) with an average size of $52 \pm 16$ $\mathrm{nm}$ (Figure 2B). To improve the tumor targetability of FPc NPs for further biomedical experiments, the colloidal surface was modified with glycol chitosan and heparin by sequential adsorption. Positively charged glycol chitosan was applied as a gluing component that adheres to the particle surface via hydrogen bonding between Pluronic and glycol units, and then attracts polyanionic heparin to the surface by electrostatic attraction [31]. Figure $2 \mathrm{C}$ shows the zeta potential alteration during the sequential adsorption. The bare FPc NPs exhibited almost neutral surface charge owing to nonionic nature of the hydrophilic PEG segment. However, the zeta potential became positive $(12.2 \pm 4.8 \mathrm{mV})$ and then changed into a negative value $(-11.6 \pm 5.1 \mathrm{mV})$ by successive addition of cationic glycol chitosan and anionic heparin. This supports the successful coating of FPc NPs with surface absorbents by layer-by-layer adsorption.

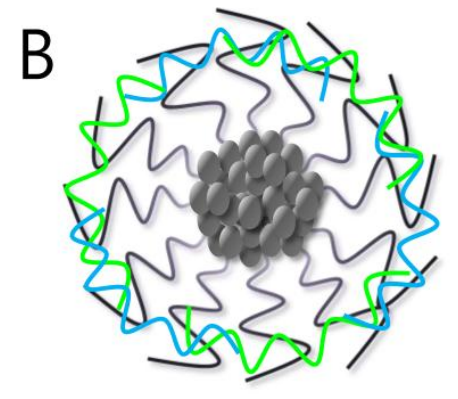

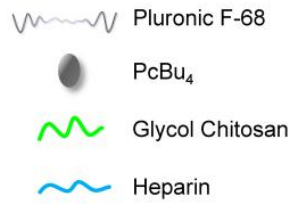

W Pluronic F-68

$\mathrm{ICMe}_{6}$

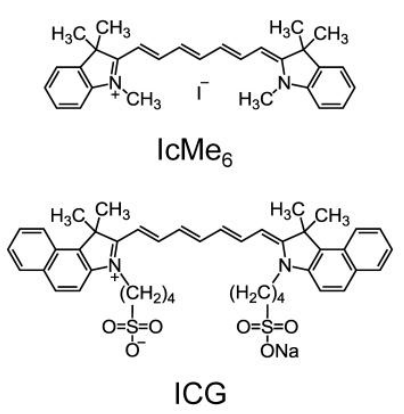

Figure I. (A) Chemical structures of $\mathrm{PcBu}_{4}$, IcMe6, and ICG. (B) Schematic representation of FPc NP.
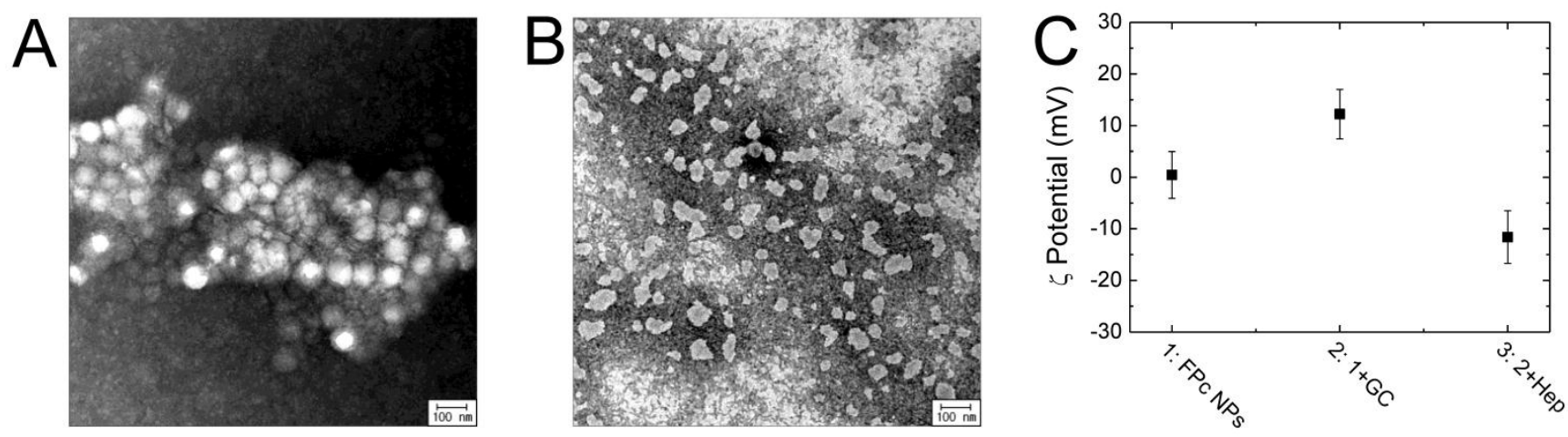

Figure 2. TEM images of FPc NPs (A) and Flc NPs (B). (C) Zeta potential values of bare FPc NPs (I) and sequentially adsorbed nanoparticles with glycol chitosan (2) and heparin (3). 

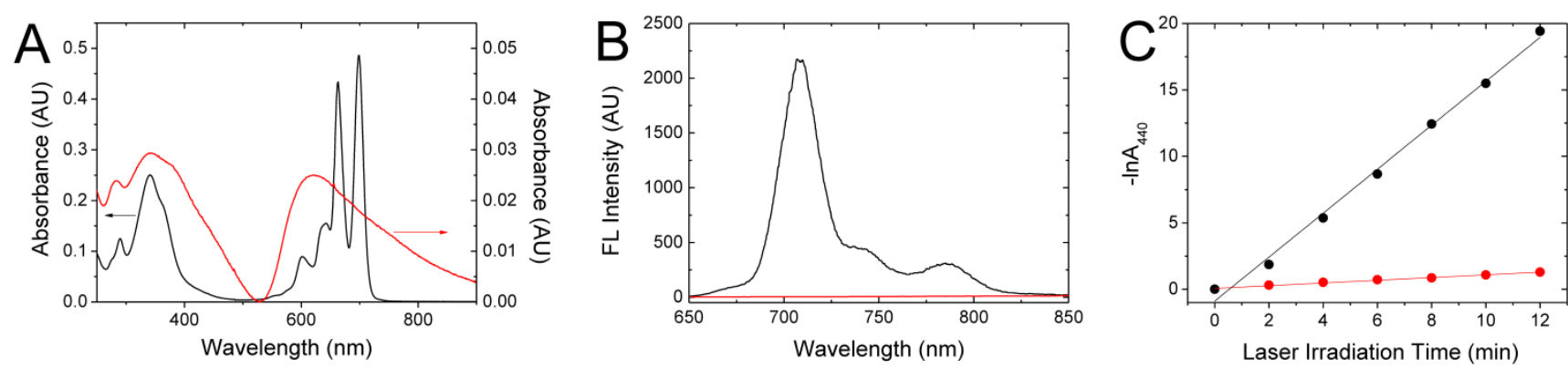

Figure 3. Absorption (A) and Fluorescence (B) spectra of FPc NPs in methylene chloride (back) and water (red) at the same concentration. (C) Photochemical bleaching of RNO by $\mathrm{IO}_{2}$ generated upon laser excitation of free chlorine e6 (Ce6) (black) and FPc NPs (red) at $67 \mathrm{I} \mathrm{nm}$, represented by the temporal dependence of RNO absorbance at $440 \mathrm{~nm}\left(-\ln A_{440}\right)$ during the irradiation of samples with the same asorbance at $67 \mathrm{Im}$ under the identical photobleaching condition. The lines are linear fits of the absorbance plots.

Figure 3 shows the optical properties of $\mathrm{PcBu}_{4}$-aggregated FPc NPs, compared to those of molecularly dispersed $\mathrm{PcBu}_{4}$. For quantitative comparison, both samples were prepared by dispersing or dissolving the thermally processed ternary mixture of FPc in water or dichloromethane at the same concentration. Free $\mathrm{PcBu}_{4}$ monomer in dichloromethane solution showed spectral fingerprints typical of the phthalocyanine chromophores [35], namely, sharp absorption bands across the visible region (a high-energy Soret transition at $341 \mathrm{~nm}$ and multiple low-energy $Q$ bands around 600-700 nm) and strong NIR fluorescence at $707 \mathrm{~nm}$. In sharp contrast, FPc NPs manifested a blunt, structureless absorption spectrum with a significant hypochromic change. The broadened $Q$ bands with an emerging longer-wavelength one at $\sim 750 \mathrm{~nm}$ (Figure $3 \mathrm{~A}$ ) are in close accordance to the spectral changes characteristic of dimeric aggregation of phthalocyanines [36, 37] . Moreover, the fluorescence of $\mathrm{PcBu}_{4}$ was completely quenched by the formation of FPc NPs (Figure 3B), as typically observed for the common organic dyes in the concentrated or aggregated state [38]. In addition to fluorescence quenching, the intersystem crossing (ISC) pathway of $\mathrm{PcBu}_{4}$ was also blocked by aggregation. It is known that the photoexcited $\mathrm{PcBu}_{4}$ in the molecularly isolated state can generate singlet oxygen $\left({ }^{1} \mathrm{O}_{2}\right)$ via ISC with the ${ }^{1} \mathrm{O}_{2}$ generation quantum yield $\left(\Phi_{\Delta}\right)$ of 0.27 [39]. The photoproduct, ${ }^{1} \mathrm{O}_{2}$, is one of reactive oxygen species (ROS) and can induce oxidative damage. However, the ${ }^{1} \mathrm{O}_{2}$ generation capability of $\mathrm{PcBu}_{4}$ was significantly depressed by its incorporation into nanoparticles at a high loading concentration $\left(\Phi_{\Delta}\right.$ $=0.04)$, as spectrally examined by the chemical oxidation of $p$-nitroso- $N, N^{\prime}$-dimethylaniline (RNO) (Figure 3C). All these manifestations evidently indicate that the crystalline $\mathrm{PcBu}_{4}$ molecules $\left(T_{\mathrm{m}}>300{ }^{\circ} \mathrm{C}\right)$ with the propensity of self-aggregation are highly aggregated inside the nanoparticulate space at the given loading content. The intraparticle aggregation can offer an optimal condition for the thermal conversion of photon energy into heat because it promotes internal conversion and vibrational relaxation of the excited state of dyes and thus efficiently blocks other competitive deactivation pathways (fluorescence decay and ISC) [40]. It is noted that absorption and fluorescence spectra of the FPc solution obtained by dissolving the thermally processed ternary mixture are identical to those of a pure $\mathrm{PcBu}_{4}$ solution without thermal treatment, evidencing that the thermally stable phthalocyanine molecules were intact without degradation during the hot mixing process (at $150{ }^{\circ} \mathrm{C}$ for $1.5 \mathrm{~h}$ ).

The photothermal heating properties were comparatively evaluated by irradiating dye-aggregated nanoparticles (FPc NPs and FIc NPs) and non-aggregated ICG solution in water with a NIR laser $\left(671 \mathrm{~nm}, 6.4 \mathrm{~W} / \mathrm{cm}^{2}\right)$. We also examined free $\mathrm{PcBu}_{4}$ solution, prepared by dissolution of thermally processed ternary mixture of FPc in toluene, to elucidate the aggregation effect on the photothermal behavior. Figure 4A shows temporal photothermal profiles measured in uncapped open containers, where the concentrations were adjusted so that the optical densities at $671 \mathrm{~nm}$ are identical for all the samples for quantitative comparison. Under irradiation, the phthalocyanine-aggregated dispersion (FPc NPs) manifested a two-phase temperature behavior: initial rapid heating above $55^{\circ} \mathrm{C}$ and then saturation after 3 min of irradiation. In the latter phase, reflux of water was observed on the container wall, suggesting that boiling occurred in the laser irradiated volume. No photothermal heating effect was observed from laser-transparent pure water without nanoparticles, clearly evidencing that the photon energies absorbed by FPc NPs were efficiently converted to molecular vibration and caused heating of the surrounding medium. In contrast to FPc NPs, other samples (FIc NPs, 
ICG and free $\mathrm{PcBu}_{4}$ ) under continuous laser irradiation did not exhibit sustained photothermal heating of the media but rather displayed temperature drops after initial heat generation. The temperature declination after initial heating is attributable to the chemical degradation of cyanine dyes under the condition with intense laser excitation and localized heat generation. To attain the sustained heat generation, thermal robustness as well as photostability might be key considerations because laser irradiation of NIR dyes is known to instantaneously raise the local temperature far above $100{ }^{\circ} \mathrm{C}$ [7]. As shown in Figure 4B, only FPC NPs retained the blue color of phthalocyanine after 15 min of laser irradiation, whereas the colors of cyanine dyes and even of free $\mathrm{PcBu}_{4}$ were completely bleached under the same condition. The comparison with cyanine-based samples evidences that phthalocyanines are highly stable optically as well as thermally, being a promising alternative to conventional cyanine dyes for the applications requiring intense light illumination. Another important point is that FPc NPs showed the greatly enhanced photostability over free $\mathrm{PcBu}_{4}$. This can be attributed to significant blocking of the oxygen-involved photobleaching process in FPc NPs, because highly aggregated $\mathrm{PcBu}_{4}$ molecules in the nanoparticulate matrix may have a low chance of contact with surrounding oxygen species. Overall, laser-excited FPc NPs generating sufficient heat to induce cellular hyperthermia validate the concept of phthalocyanine nanoaggregation to produce high-performance organic NIR absorbers for PTT.

The above advantageous photothermal effect prompted us to explore the possible utility of FPc NPs as a PTT agent. First, the tumor targeting behavior was examined in cells and in vivo. For monitoring by fluorescence imaging, the GC amines on the nanoparticle surface were labeled with Cy5.5 since its absorption and fluorescence are located in the tissue-penetrating near-infrared (NIR) spectral window $[1,2]$. Figure 5A demonstrates the in vitro potential of FPc NPs to target live cancer cells. When FPc NPs were incubated with SCC7 (squamous cell carcinoma) cells for $1 \mathrm{~h}$, clear NIR fluorescence (NIRF) signals were observed from the intracellular regions. This indicates that FPc NPs are cell membrane-permeable to attain intracellular accumulation, which is a prerequisite condition for efficient hyperthermia of cancer cells. More importantly, FPc NPs also presented the efficient tumor targeting capability in vivo. Figure $5 \mathrm{~B}$ shows noninvasive visualization of the pharmacokinetic behavior: FPc NPs were systemically administered into SCC7 tumor-bearing mice via tail vein injection and the NIRF images were taken with the eXplore Optix system at selected time points after intravenous injection. Immediately after injection (10 min), FPc NPs displayed strong NIRF signal throughout the injected body. This implies good blood circulation of the Pluronic-based nanoparticles with minimal RES filtration in liver or spleen, presumably due to the antifouling surface nature arising from the PEG segment. It is noted that a marked image contrast was only seen at the tumor. Importantly, the tumor signal was further increased in the course of time, while the whole-body signal was gradually decreased: at $6 \mathrm{~h}$ post-injection, FPc NPs were mainly taken up by the tumor so as to readily identify the tumor location. This behavior indicates significant tumor accumulation of the blood-circulating Pluronic nanoparticles thanks to the size-motivated EPR effect. All the imaging results conclude that the photothermal FPc NPs have great tumor targetability in vitro as well as in vivo, suggesting the possible utility in PTT application.

The photothermally induced toxicity was then evaluated by cell viability (MTT) assay [41] with live SCC7 cells with and without treatment of FPc NPs (Figure 6A). To induce the phototoxic effect, cells were treated with a laser $\left(671 \mathrm{~nm}, 6.4 \mathrm{~W} / \mathrm{cm}^{2}\right)$ for varying time of irradiation. Without phototreatment, FPc NPs showed minimal dark toxicity, probably due to the biocompatible surface nature and tiny colloidal size. Under laser illumination, the phototoxicity was clearly seen in nanoparticle-treated cells, but not in sample-free control cells. FPc NPs-treated cells showed a gradual decline in the cell viability with increasing dose of light. This result suggests that the photothermally generated heat by cell-internalized FPc NPs imposes a significant impact on the cell viability to induce hyperthermia. It should be noted that the photosensitization of cytotoxic ${ }^{1} \mathrm{O}_{2}$ by FPc NPs is possible as discussed in Figure 3C but cannot be a major cause of the observed phototoxic effect since its efficiency is highly depressed due to the aggregation-induced blocking of ISC. The phototherapeutic effect was further assessed in vivo with SCC7 tumor-bearing mice that were administered with FPc NPs via tail vein injection. Referring to the in vivo imaging result (Figure 5B), a NIR laser $(671 \mathrm{~nm}, 6.4$ $\mathrm{W} / \mathrm{cm}^{2}, 2 \mathrm{~mm}$ in beam diameter) was scanned throughout the tumor at $4 \mathrm{~h}$ post-injection (each scanning spot was irradiated for $3 \mathrm{~min}$ ). Figure $6 \mathrm{~B}$ shows the comparative tumor growth data for laser-treated mice with and without pre-administration of FPc NPs. Without injection of photothermal nanoparticles, no notable therapeutic effect was observed: the tumor kept growing in the course of time after laser treatment. In sharp contrast, the mice that were administered with both FPc NPs and light manifested 
significantly suppressed tumor growth compared to the FPc NPs-free control. Overall, the light-activated cytotoxicity and apparent suppression of tumor growth elucidate that phthalocyanine-aggregated all-organic NIR nanoabsorbers have potential as a nanomedicine for photothermally induced hyperthermia of cancer.
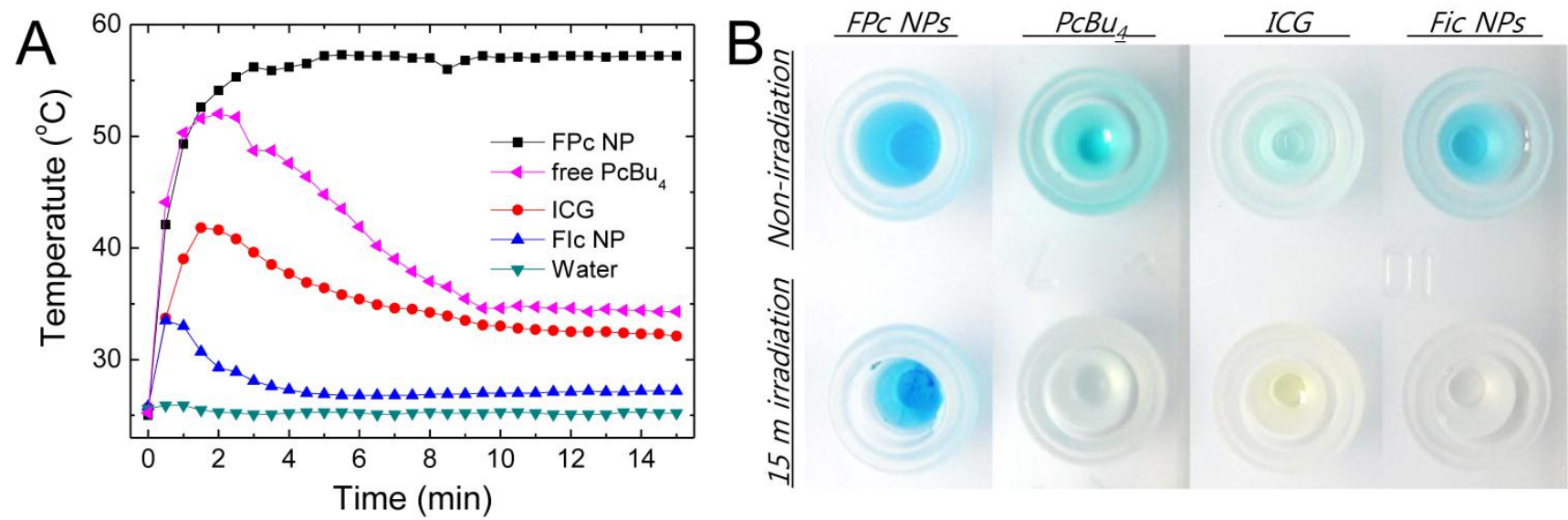

Figure 4. (A) Temperature evolution in suspensions of FPc NPs and Flc NPs, free PcBu4 (prepared by dissolving the dried ternary mixture of FPc in toluene), water solution of ICG, and pure water during continuous irradiation of $67 \mathrm{I} \mathrm{nm}$ laser at $6.4 \mathrm{~W} / \mathrm{cm}^{2} \mathrm{for} 15 \mathrm{~min}$. (B) Image of sample-containing wells before and after laser irradiation.
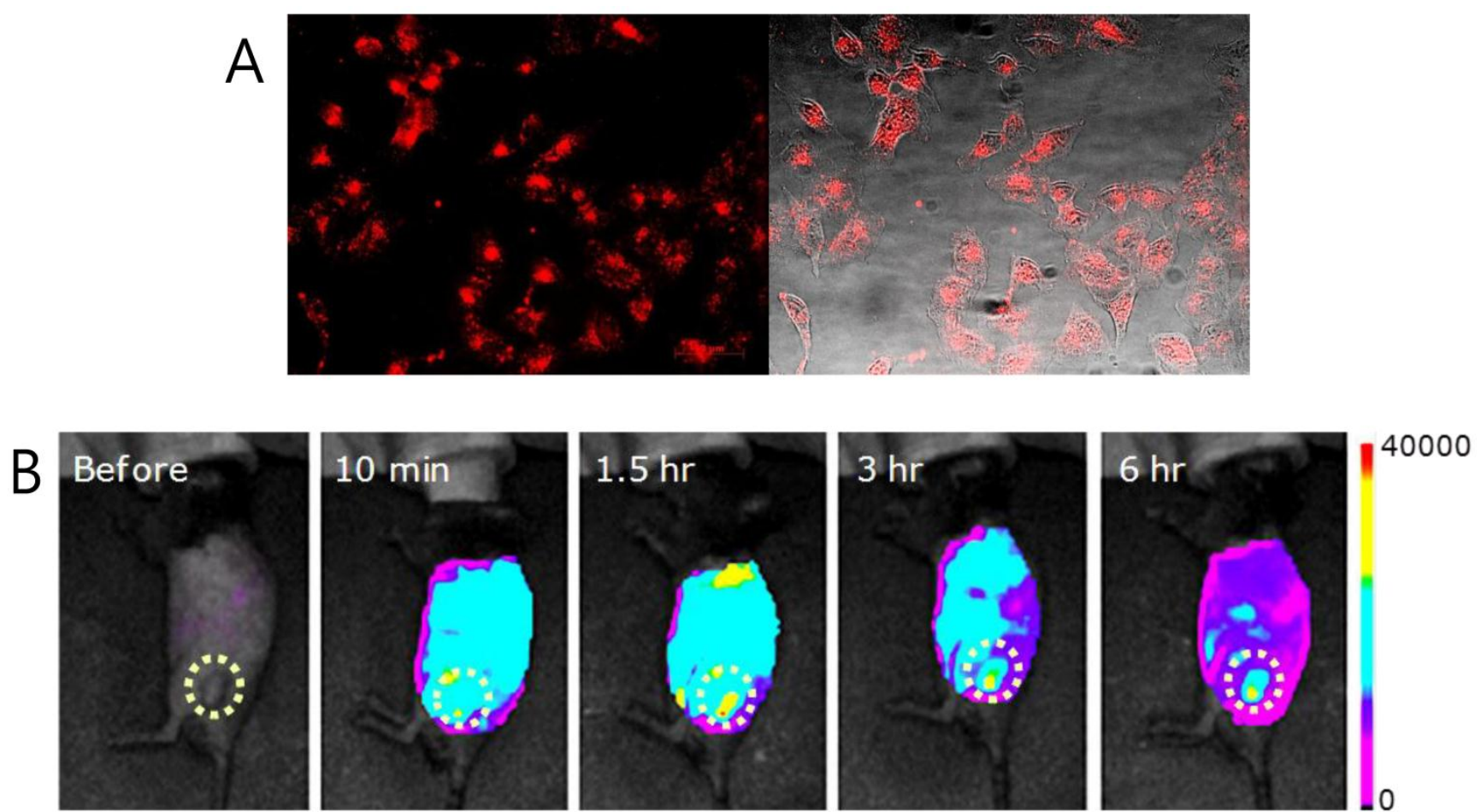

Figure 5. (A) Fluorescence (left) and optically merged (right) images of SCC7 cells treated with Cy5.5 labeled FPc NPs. (B) In vivo NIRF images of SCC7 tumor-bearing mouse before and after tail vein injection of Cy5.5 labeled FPc NPs (I00 $\mu \mathrm{L} \mathrm{of} \mathrm{Img/mL} \mathrm{FPc} \mathrm{NPs).} \mathrm{Imaging}$ time points after injection are indicated. 

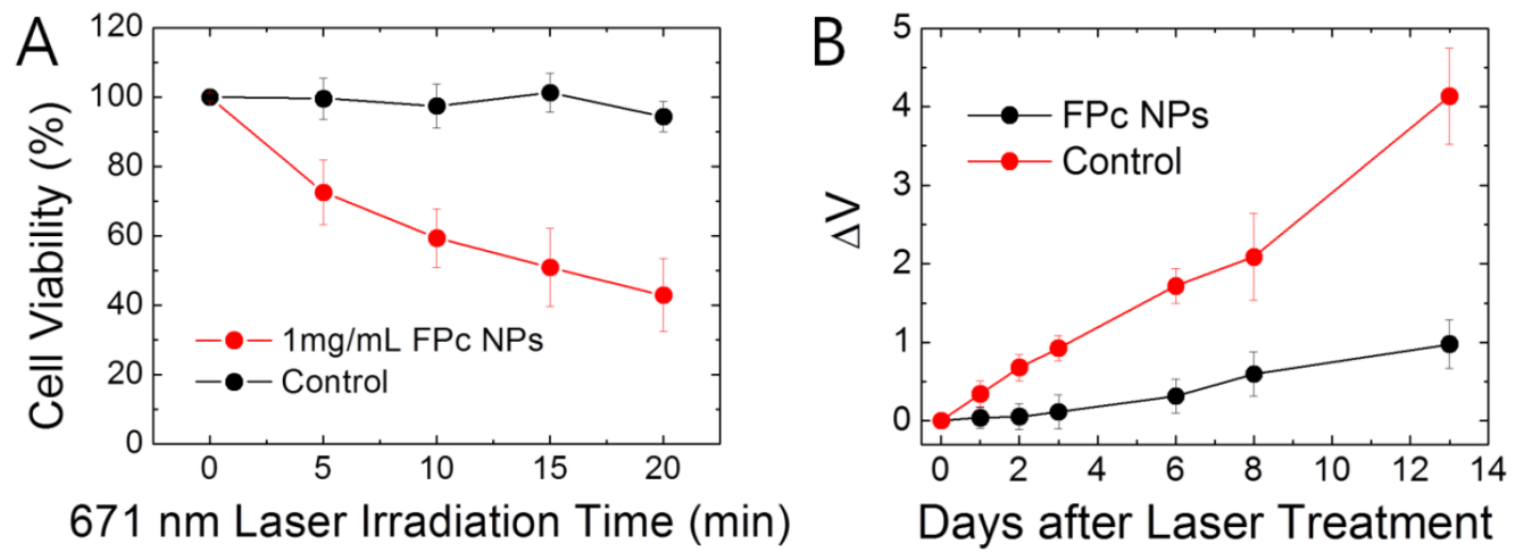

Figure 6. (A) Viability of laser-exposed SCC7 cells without (black) or with (red) treatment of FPc NPs as a function of laser irradiation time. The data were collected from MTT assay at $12 \mathrm{~h}$ after the laser exposure. The error bars indicate the standard deviations for independent experiments $(n=10)$. (B) Tumor growth $(\Delta V)$ of laser-exposed SCC7 tumor-bearing mice with or without (control) intravenous injection of FPc NPs $(200 \mu \mathrm{L}$ of I mg mL-1 FPc NPs). The error bars indicate the standard deviations for independent experiments $(n=4)$.

\section{Conclusion}

We have formulated Pluronic-based NIR-abosrbing nanoparticles (FPc NPs) whose interior is concentrated with a hydrophobic phthalocyanine dye. Owing to the outstanding optical and thermal stability of $\mathrm{PcBu}_{4}$, as well as aggregation-promoted thermal relaxation, FPc NPs presented robust photothermal effect superior to an individual cyanine dye and cyanine-aggregated nanoparticles. In addition, FPc NPs exhibited excellent cancer targetability in vitro and in vivo, thanks to the mesoscopic colloidal size and biologically favorable surface characteristics arising from the biopolymeric adsorbents. By virtue of these combined merits, FPc NPs demonstrated possible utility as an all-organic NIR nanoabsorber for photothermal therapy of cancer.

\section{Acknowledgment}

This work was supported by grants from the Korea Ministry of Education, Science and Technology (MEST) (Nos. 2012-0001082 and 2012-0006061) and by the Intramural Research Program of KIST.

\section{Competing Interests}

The authors have declared that no competing interest exists.

\section{References}

1. Prasad PN. Introduction to Biophotonics. Hoboken, New Jersey: John Wiley \& Sons. 2003.

2. Weissleder R, Ntziachristos V. Shedding light onto live molecular targets. Nat. Med. 2003;9:123-128.

3. Dolmans DEJGJ, Fukumura D, Jain RK. Photodynamic therapy for cancer. Nature Reviews Cancer. 2003;3:380-387.

4. Marchant AB. Optical Recording. New York: Addison-Wesley. 1990.
5. Irie M. Photo-reactive Materials for Ultrahigh Density Optical Memory. Amsterdam: Elsevier. 1994.

6. Matsuzawa N, Seto J. Effect of the Blending of Binder Polymers on Recording Sensitivity of Dye/Polymer Optical Recording Media. Jpn. J. Appl. Phys. 1991;30:L1770-L1773.

7. Huh YJ, Kim SH, Kim SC. Thermal Decomposition and Deformation of Dye and Polycarbonate in Compact Disc-Recordables. Jpn. J. Appl. Phys. 1997;36:7233-7238.

8. Suzuki Y, Okamoto Y, Kurose Y, Maeda S. High-Speed Recording Performance of Metal Azo Dye Containing Digital Video Disc-Recordable Discs. Jpn. J. Appl. Phys. 1999;38:1669-1674.

9. Fujii T, Tajima T, Negishi R, Okitsu I, Tomizawa Y, Matsuda I, Hamada E. Approach for High Speed Recording of 4.7 GB Digital Versatile Disc-Recordable. Jpn. J. Appl. Phys. 2000;39:779-784.

10. Chen WR, Adams RL, Bartels KE, Nordquist RE. Chromophore-enhanced in vivo tumor- cell destruction using an $808-\mathrm{Nm}$ diode-laser. Cancer Lett. 1995;94:125-131.

11. Chen WR, Adams RL, Higgins AK, Bartels KE, Nordquist RE. Photothermal effects on murine mammary tumors using indocyanine green and an 808-nm diode laser: an in vivo efficacy study. Cancer Lett. 1996;98:169-173.

12. Nolsoe CP, Torppedersen S, Burcharth F, Horn T, Pedersen S, Christensen NEH, Olldag ES, Andersen PH, Karstrup S, Lorentzen T, Holm HH. Interstitial hyperthermia of colorectal liver metastases with a US-guided Nd-YAG laser with a diffuser tip: a pilot clinical study. Radiology. 1993;187:333-337.

13. Sultan RA. Tumour ablation by laser in general surgery. Lasers Med. Sci. 1990;5:185-193.

14. Kam NWS, O'Connell M, Wisdom JA, Dai H. Carbon nanotubes as multifunctional biological transporters and near-infrared agents for selective cancer cell destruction. Proc. Natl. Acad. Sci. U.S.A. 2005;102:11600-11605.

15. Chakravarty P, Marches R, Zimmerman NS, Swafford AD-E, Bajaj P, Musselman IH, Pantano P, Draper RK, Vitetta ES. Thermal ablation of tumor cells with antibody-functionalized single-walled carbon nanotubes. Proc. Natl. Acad. Sci. U.S.A. 2008;105:8697-8702.

16. Ghosh S, Dutta S, Gomes E, Carroll D, D'Agostino JrR, Olson J, Guthold M, Gmeiner WH. Increased Heating Efficiency and Selective Thermal Ablation of Malignant Tissue with DNA-Encased Multiwalled Carbon Nanotubes. ACS Nano 2009;3:2667-2673.

17. Moon HK, Lee SH, Choi HC. In Vivo Near-Infrared Mediated Tumor Destruction by Photothermal Effect of Carbon Nanotubes. ACS Nano 2009;3:3707-3713.

18. Hirsch LR, Stafford RJ, Bankson JA, Sershen SR, Rivera B, Price RE, Hazle JD, Halas NJ, West JL. Nanoshell-mediated near-infrared thermal therapy of tumors under magnetic resonance guidance. Proc. Natl. Acad. Sci. U.S.A. 2003;100:13549-13554. 
19. Huang X, El-Sayed IH, Qian W, El-Sayed MA. Cancer Cell Imaging and Photothermal Therapy in the Near-Infrared Region by Using Gold Nanorods. J. Am. Chem. Soc. 2006;128:2115-2120.

20. Gobin AM, Lee MH, Halas NJ, James WD, Drezek RA, West JL. Near-Infrared Resonant Nanoshells for Combined Optical Imaging and Photothermal Cancer Therapy. Nano Lett. 2007;7:1929-1934.

21. Skrabalak SE, Au L, Lu XM, Li XD, Xia YN. Gold nanocages for cancer detection and treatment. Nanomedicine 2007;2:657-668.

22. Zhang JZ. Biomedical Applications of Shape-Controlled Plasmonic Nanostructures: A Case Study of Hollow Gold Nanospheres for Photothermal Ablation Therapy of Cancer. J. Phys. Chem. Lett. 2010;1:686-695.

23. Resch-Genger U, Grabolle M, Cavaliere-Jaricot S, Nitschke R, Nann T. Quantum dots versus organic dyes as fluorescent labels. Nat. Methods. 2008;5:763-775.

24. Maeda H, Wu J, Sawa T, Matsumura Y, Hori K. Tumor vascular permeability and the EPR effect in macromolecular therapeutics: a review. J. Control. Release. 2000;65:271-284.

25. Gordon PF, Gregory P. Organic Chemistry in Color. Berlin-Heidelberg-London: Springer-Verlag. 1983.

26. Shima T, Yamakawa $Y$, Nakano T, Kim J, Tominaga J. Metal-Free Phthalocyanine Layer Prepared on Read-Only-Memory Disc for Super-Resolution Readout. Jpn. J. Appl. Phys. 2006;45:L1007-L1009.

27. Tan JS, Butterfield DE, Voycheck CL, Caldwell KD, Li JT. Surface modification of nanoparticles by PEO/PPO block copolymers to minimize interactions with blood components and prolong blood circulation in rats. Biomaterials. 1993;14:823-833.

28. Muniruzzaman M, Marin A, Luo Y, Prestwich GD, Pitt WG, Husseini G, Rapoport NY. Intracellular uptake of Pluronic copolymer: effects of the aggregation state. Coll. Surf. B-Biointerf. 2002;25:233-241.

29. Mei L, Zhang Y, Zheng Y, Tian G, Song C, Yang D, Chen H, Sun H, Tian Y, Liu K, Li Z, Huang L. A Novel Docetaxel-Loaded Poly ( $\varepsilon$-Caprolactone)/Pluronic F68 Nanoparticle Overcoming Multidrug Resistance for Breast Cancer Treatment. Nanoscale Res. Lett. 2009;4:1530-1539.

30. Yuk SH, Oh KS, Cho SH, Lee BS, Kim SY, Kwak BK, Kim K, Kwon IC. Glycol Chitosan/Heparin Immobilized Iron Oxide Nanoparticles with a Tumor-Targeting Characteristic for Magnetic Resonance Imaging. Biomacromolecules. 2011;12:2335-2343.

31. Yuk SH, Oh KS, Cho SH, Kim SY, Oh S, Lee JH, Kim K, Kwon IC. Enhancement of the Targeting Capabilities of the Paclitaxel-Loaded Pluronic Nanoparticles with a Glycol Chitosan/Heparin Composite. Mol. Pharmaceutics. 2012;9:230-236.

32. Lee KD, Nir S, Papahadjopoulos D. Quantitative analysis of liposome-cell interactions in vitro: rate constants of binding and endocytosis with suspension and adherent J774 cells and human monocytes. Biochemistry 1993, 32: 889-899.

33. Davda J, Labhasetwar V. Characterization of nanoparticle uptake by endothelial cells. Int. J. Pharm. 2002;233:51-59.

34. Nie SM, Xing Y, Kim GJ, Simons JW. Nanotechnology applications in cancer. Annu. Rev. Biomed. Eng. 2007;9:257-288.

35. Guldi DM, Zilbermann I, Gouloumis A, Vázquez P, Torres T. Metallophthalocyanines: Versatile Electron-Donating Building Blocks for Fullerene Dyads. J. Phys. Chem. B. 2004;108:18485-18494.

36. Snow AW. In The Porphyrin Handbook; Kadish KM, Smith KM, Guilard R, Eds. Amsterdam: Elsevier Science, 2003;109-129.

37. Ranade A, Hiltner A, Baer E, Shirk JS, Lepkowicz RS. Aggregation of Lead Phthalocyanine in Blends with Polycarbonate. J. Appl. Polym. Sci. 2007:104:464-469.

38. Birks JB. Photophysics of Aromatic Molecules. London: Wiley. 1970.

39. Tanielian C, Wolff C. Porphyrin-Sensitized Generation of Singlet Molecular Oxygen: Comparison of Steady-State and Time-Resolved Methods. J. Phys. Chem. 1995;99:9825-9830.

40. Lovell JF, Jin CS, Huynh E, Jin H, Kim C, Rubinstein JL, Chan WCW, Cao W, Wang LV, Zheng G. Porphysome nanovesicles generated by porphyrin bilayers for use as multimodal biophotonic contrast agents. Nat. Mater. 2011;10:324-332.

41. Mosmann T. Rapid colorimetric assay for cellular growth and survival: application to proliferation and cytotoxicity assays. J. Immunol. Methods 1983;65:55-63. 